\title{
Response to nivolumab combining radiotherapy and nimotuzumab in metastatic oral squamous cell carcinoma patient with strong PD-L1 expression: a case report
}

\author{
Xiao Tang ${ }^{1}$, Shiqing Chen ${ }^{2}$, Qianqian Sui ${ }^{2}$, Xiaoguang Li ${ }^{1}$, Zhonglong Liu ${ }^{1}$, Fengshuo Zhu ${ }^{1}$, Jiping Ding ${ }^{3}$, \\ Yuan $\mathrm{Yao}^{3}$, Bin Jiang ${ }^{4}$, Yue $\mathrm{He}^{1}$ \\ ${ }^{1}$ Department of Oral and Maxillofacial-Head and Neck Oncology, Shanghai Ninth People's Hospital, College of Stomatology, Shanghai Jiao Tong \\ University School of Medicine, Shanghai 200011, China; ${ }^{2}$ The Medical Department, 3D Medicines Inc., Shanghai 201114, China; ${ }^{3}$ Department of \\ Oncology radiotherapy, ${ }^{4}$ Oncology Department, Shanghai Ninth People's Hospital, Shanghai Jiao Tong University School of Medicine, Shanghai \\ 200011, China \\ Correspondence to: Yue He. Department of Oral and Maxillofacial-Head and Neck Oncology, Shanghai Ninth People's Hospital, College of \\ Stomatology, Shanghai Jiao Tong University School of Medicine, 639 Zhi Zao Ju Road, Shanghai 200011, China. Email: william5218@126.com.
}

\begin{abstract}
Immune checkpoint inhibitors (ICIs) have been approved for the treatment of oral squamous cell carcinoma (OSCC). However, not all patients would benefit from ICIs. Thus, in order to improve the response rate, the efficacy and safety of combination immunotherapy is still under evaluation. We report a 71-year-old female patient with a recurrent metastatic oral cavity cancer. Immunohistochemical examination showed more than $50 \%$ of tumor cells to express the programmed cell death receptor ligand 1 (PD-L1). The patient received nivolumab plus radiotherapy and nimotuzumab. Both clinically and radiologically response was observed and the patient reached a partial response (PR). This is the first case describing the efficacy and safety of nivolumab plus radiotherapy and nimotuzumab in an OSCC patient with a strong PD-L1 expression, showing a tolerable safety profile and a promising response. The combination immunotherapy may be a potential treatment option for patients with OSCC.
\end{abstract}

Keywords: Combination immunotherapy; programmed cell death receptor ligand 1 (PD-L1); oral squamous cell carcinoma (OSCC); case report

Submitted Oct 31, 2019. Accepted for publication Feb 10, 2020.

doi: $10.21037 /$ atm.2020.02.96

View this article at: http://dx.doi.org/10.21037/atm.2020.02.96

\section{Introduction}

Oral squamous cell carcinoma (OSCC) is one type of head and neck cancer, accounting for approximately $1.12 \%$ new cases and $0.79 \%$ mortalities of all cancers in China (1). The age-standardized 5 -year relative survival of Oral cavity and pharynx cancer $50.4 \%$ in China (2). Therapeutic options are limited for metastasis OSCC, with poor prognosis. Standard therapy of recurrent and metastatic head and neck cancer is cisplatin, 5-fluorouracil, cetuximab, or a combination of these agents, with a median overall survival (OS) of 610 months (3-5). Immune checkpoint inhibitors (ICIs) such as programmed cell death 1 (PD-1)/programmed deathligand 1 (PD-L1) inhibitors treatment, have demonstrated a clear survival benefit in various tumor types, including nonsmall cell lung cancer (NSCLC), bladder cancer, gastric or gastroesophageal junction cancer, renal cell carcinoma, breast cancer, melanoma, and head and neck squamous cell carcinoma (HNSCC). Several clinical trials assessing ICI in HNSCC obtained promising results in second-line or firstline therapy, such as CheckMate 141, KEYNOTE-040, and KEYNOTE-048 (4-6). In 2016, the US Food and Drug Administration (FDA) approved pembrolizumab and nivolumab as a second-line therapy for the treatment of HNSCC. However, in unselected patients with HNSCC, objective response rate (ORR) of second-line immunotherapy was only $13.3-14.6 \%$, whereas patients with a positive PD- 


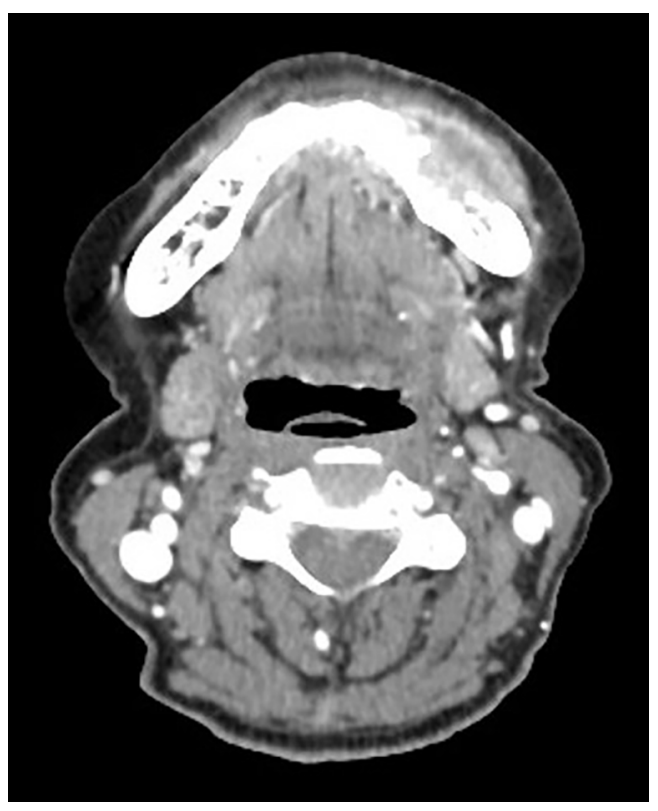

Figure $1 \mathrm{CT}$ of the primary tumor. Contrast-enhanced CT showed a $3 \mathrm{~cm} \times 1.7 \mathrm{~cm}$ mass of the left mandible. CT, computed tomography.

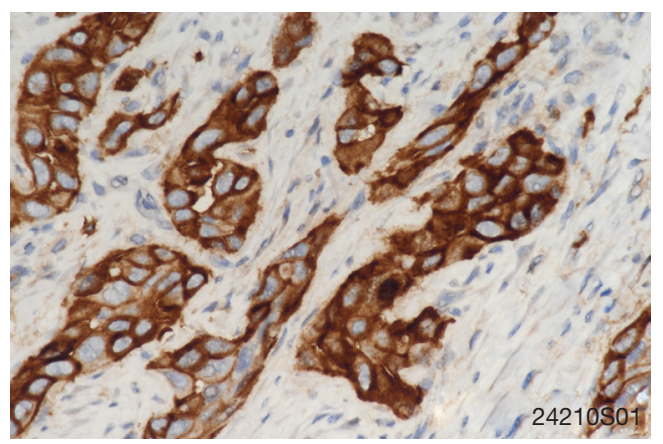

Figure 2 The patient was PD-L1 strong expression. Immunohistochemical staining of PD-L1 expression $(\times 400)$. The PD-L1 expression of patient sample was $50 \%$. PD-L1 was stained by VENTANA PD-L1 (SP263) Assay kit following manufactures' instructions. Positive control and negative control were provided by the kit, indicating PD-L1 expressing and non-PD-L1 expressing tissues, respectively. The "patient sample" was paraffin section of FFPE embedded tumor sample. PD-L1, programmed cell death receptor ligand 1 .
L1 expression obtained higher ORR.

Nowadays, data of clinical trials of immunotherapies in Chinese HNSCC patients are insufficient. Potential predictors of drug efficacy are also in urgent need of further exploration. Herein, we report a recurrent metastatic oral cavity cancer patient with strong PD-L1 expression ( $\geq 50 \%$ ) who obtained significant response to nivolumab treatment. We present the following case in accordance with the CARE Guideline (7).

\section{Case presentation}

A 71-year-old female patient was initially admitted to Shanghai Ninth People's Hospital in February 2018 with soft tissue swelling of the left mandible. She represented no history or family history of cancer, without pathogenic germline mutation. Contrastenhanced computed tomography (CT) showed a $3 \mathrm{~cm} \times$ $1.7 \mathrm{~cm}$ mass (Figure 1). Postoperative pathology indicated Grade I-II squamous cell carcinoma. Two months after the excision of the left mandible, CT showed tumor recurrence in the left bottom of oral cavity. The patient received operation again and treatment with tegafur, gimeracil and oteracil potassium capsules (TGOP). On $25^{\text {th }}$ July 2018, positron emission tomography-CT (PET-CT) showed multiple metastases, involving tongue, oropharynx, submandibular gland, parotid gland, both sides of the neck and left supraclavicular lymph nodes. We subsequently performed extended resection for swelling of the left mandible, bilateral lymph node dissection and tracheotomy. On $31^{\text {st }}$ August 2018, follow-up CT revealed multiple lung metastases.

To seek other potential therapeutic opportunities, the patient received next generation sequencing (NGS) analysis using a 381-gene panel in a College of American Pathologists (CAP) certificated lab (3D Medicines Inc., Shanghai, China) and immunohistochemistry (IHC) detection of PD-L1 expression. NGS results indicated that the tumor mutation burden (TMB) was 0.81 Mutants/Mb and the microsatellite state was stable. IHC results indicated that more than $50 \%$ tumor cells expressed the PD-L1 
(Figure 2). TMB was defined as the number of somatic mutations per million bases in sequenced coding region. No other immunotherapy or targeted therapy related biomarkers were identified (Table 1).

According to NCCN guidelines and the results from the two large clinical trials (CheckMate 141 and KEYNOTE-040), immunotherapy was considered. The patient received nivolumab treatment $(100 \mathrm{mg}$, intravenously every 2 weeks) since September, 2018. Even though the PD-L1 expression was high, the TMB was relatively low, thus radiotherapy and nimotuzumab were additionally given to the patient at the first two cycles of nivolumab treatment. The prescription dose to the gross target volume (GTV) was $66 \mathrm{~Gy} / 30 \mathrm{f}$ daily, and clinical target volume (CTV) was $54 \mathrm{~Gy} / 30 \mathrm{f}$ daily for 1 month. Nimotuzumab was administered at a dose of $200 \mathrm{mg}$ every week for 1 month (for details of the course see Figure 3). Lesions in lung received significant response to the

Table 1 Results of NGS

\begin{tabular}{lc}
\hline Items & Result \\
\hline TMB (Mutants/Mb) & 4.03 \\
Microsatellite & Stable \\
Gene mutation & CYP2C19 (rs4244285*2, c.681G $>$ A) \\
\hline
\end{tabular}

NGS, next generation sequencing; TMB, tumor mutation burden. treatment (Figure 4). PET-CT showed that the metabolic activity of cancer cells in multiple lesions was reduced (Figure 5). At the time of this writing, the patient is still alive with stable, asymptomatic disease and the progressionfree survival (PFS) was more than 10 months. During the combination treatment, the patient presented grade 1 pneumonitis, and the symptom was relieved after the administration of imipenem and cilastatin sodium.

\section{Discussion and conclusions}

In this case, the OSCC patient with strong PD-L1 expression $(\geq 50 \%)$ responded deeply to nivolumab plus radiotherapy and nimotuzumab. To our knowledge, this is the first case presenting response to ICI combining with radiotherapy and nimotuzumab in metastasis OSCC in China.

PD-L 1 expression has been considered as an immunotherapy biomarker in NSCLC, melanoma, urothelial carcinoma and HNSCC, and the expression level was associated with response (6,8-10). In CheckMate 141 trial, ORR in nivolumab arm was $17.0 \%, 22.2 \%$, $27.9 \%$ respectively in HNSCC patients with PD-L1 expression $\geq 1 \%, \geq 5 \%, \geq 10 \%$, whereas the patients with negative PD-L1 expression ( $<1 \%)$ had an ORR of $12.3 \%$. In KEYNOTE-040, the ORR reached $26.6 \%$ in HNSCC patients with strong PD-L1 expression $(\geq 50 \%)$ treated

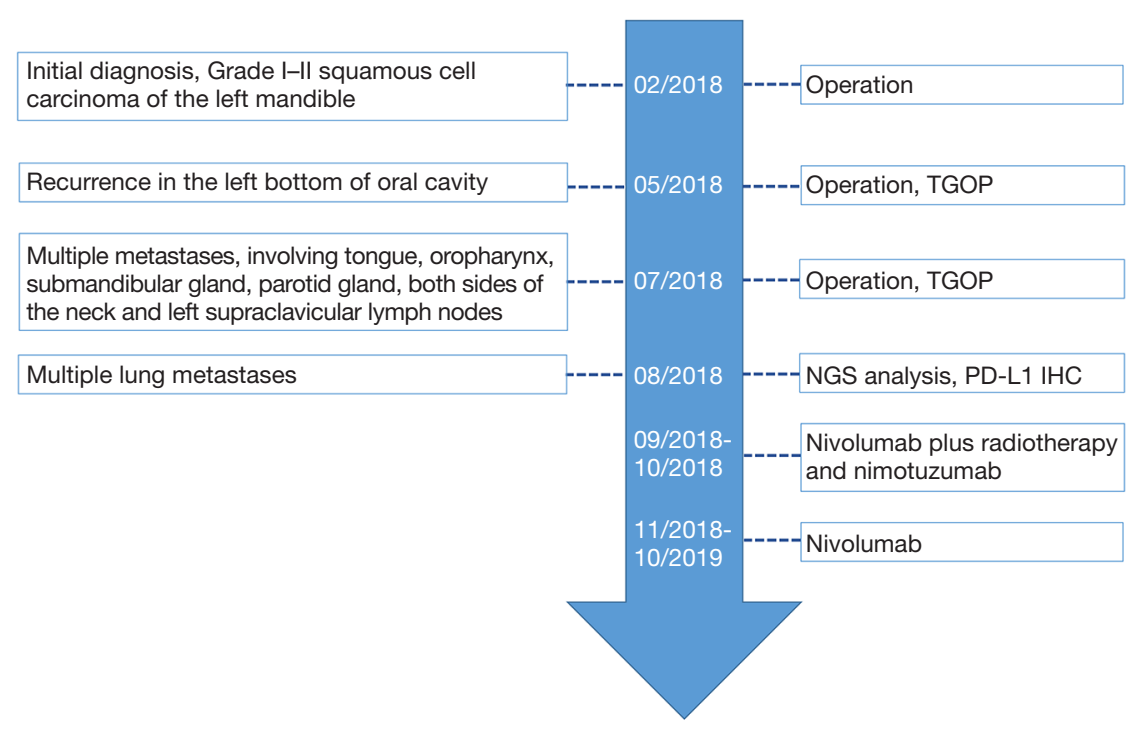

Figure 3 Timeline of diagnosis and treatment. PD-L1, programmed cell death receptor ligand 1; IHC, immunohistochemistry; NGS, next generation sequencing; TGOP, tegafur, gimeracil and oteracil potassium capsules. 

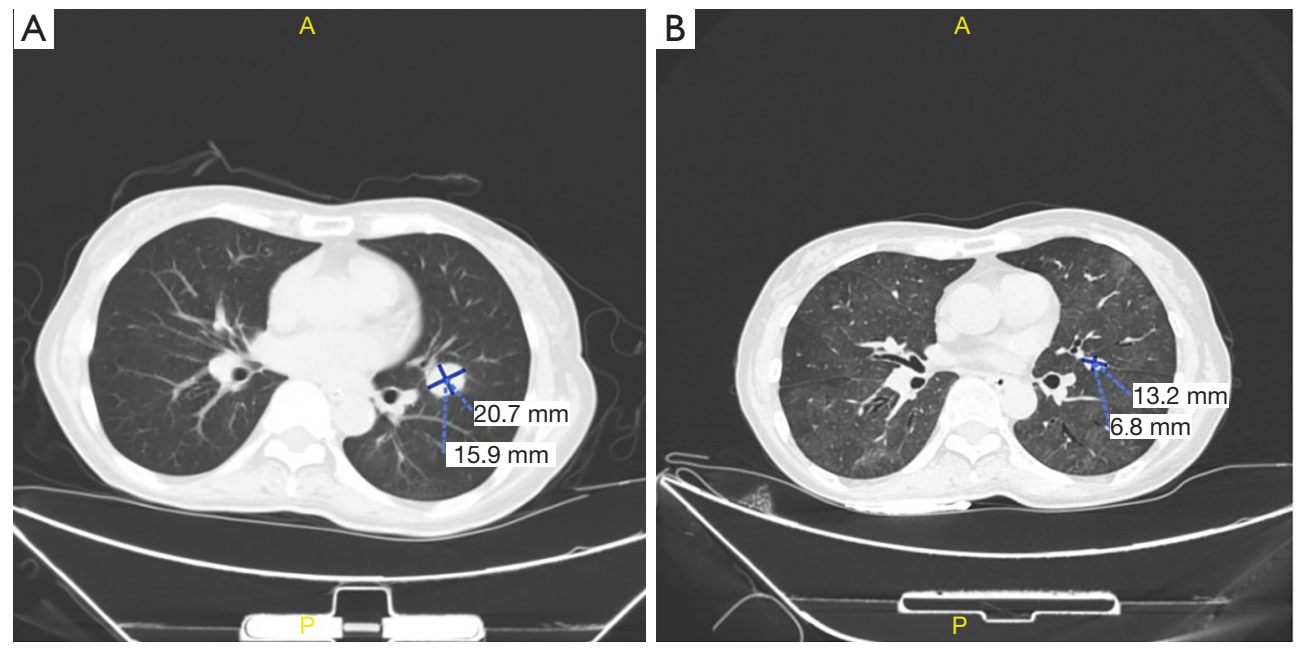

Figure 4 CT scan shows different lesions in lung during immunotherapy. (A) Lesions before immunotherapy were indicated by arrows; (B) lesions after immunotherapy were indicated by arrows. CT, computed tomography.

with Pembrolizumab as a second-line therapy. More recently, FDA has approved pembrolizumab as a firstline therapy for patients with metastatic or recurrent and inoperable HNSCC with positive PD-L1. KEYNOTE-048 trial revealed Pembrolizumab monotherapy as a first-line therapy didn't significantly improve OS compared with chemotherapy in the total population (HR: 0.83 , 95\% CI: 0.70-0.99, $\mathrm{P}=0.0199$; median 11.5 vs. $10.7 \mathrm{mo}$ ), whereas significantly improved OS in the CPS $\geq 20$ population (HR: $0.58,95 \%$ CI: 0.44-0.78; median 14.8 vs. 10.7 mo). What's more, ICI monotherapy exhibited more favorable safety profile than chemotherapy, with less treatmentrelated adverse events, especially grade 3-5 adverse events. Besides PD-L1 expression, TMB is also approved to be a biomarker for ICIs. In clinical trial CHECKMATE 026 and CHECKMATE 227, compared with TMB-middle and TMB-low patients, TMB-high subpopulation received better clinical outcomes $(11,12)$. In this case, PD-L1 expression was strong, while TMB was relatively low, so we applied nivolumab with radiotherapy plus nimotuzumab to treat the patient and the patient achieved a partial response (PR).

A combination of ICI and other therapy has revealed better efficacy to cancer patients. According to a secondary analysis of KEYNOTE-001 trial and other clinical evidences, previous radiotherapy may enhance PFS and OS in patients receiving immunotherapy (13). This is presumably because that tumor cells damaged by radiotherapy could release antigens that could serve as adaptive immune response targets and enhance immune response. To date, a large number trials evaluating the combination of ICI with radiation are ongoing, most of which are early-phase trials focusing on the safety (14). Furthermore, ICI incorporating with antiangiogenic agents exhibited surprising outcome in multiple tumor types, such as renal cell carcinoma and hepatocellular carcinoma $(15,16)$.

In this case, NGS analysis showed the patient had no potential biomarker associated with HPD, and PDL1 expression was strong ( $\geq 50 \%)$, so treatment with ICI was considered reasonable. Combination of ICI with radiotherapy and antiangiogenic agent may prevent the patient from hyperprogressive disease (HPD), which may be a novel tumor response pattern in patients treated by ICI (17-20). HPD was observed in $29 \%$ of patients with HNSCC (21). Therefore, we applied nivolumab with radiotherapy plus Nimotuzumab to treat the patient. In our case, the TMB was low and the microsatellites were stable, thus we speculated that strong PD-L1 expression is the main reason for the deep response. This is a single case, and we can't comprehensively understand how combination of ICI with radiotherapy and antiangiogenic work on OSCC, which remain to be clarified by future clinical trials.

In conclusion, this is the first case describing a strong PD-L1 positive OSCC patient who received a significant response to nivolumab treatment combining with radiotherapy and nimotuzumab. This case indicated that ICI combination therapy may bring new treatment option for patients with recurrent or metastatic OSCC, especially 

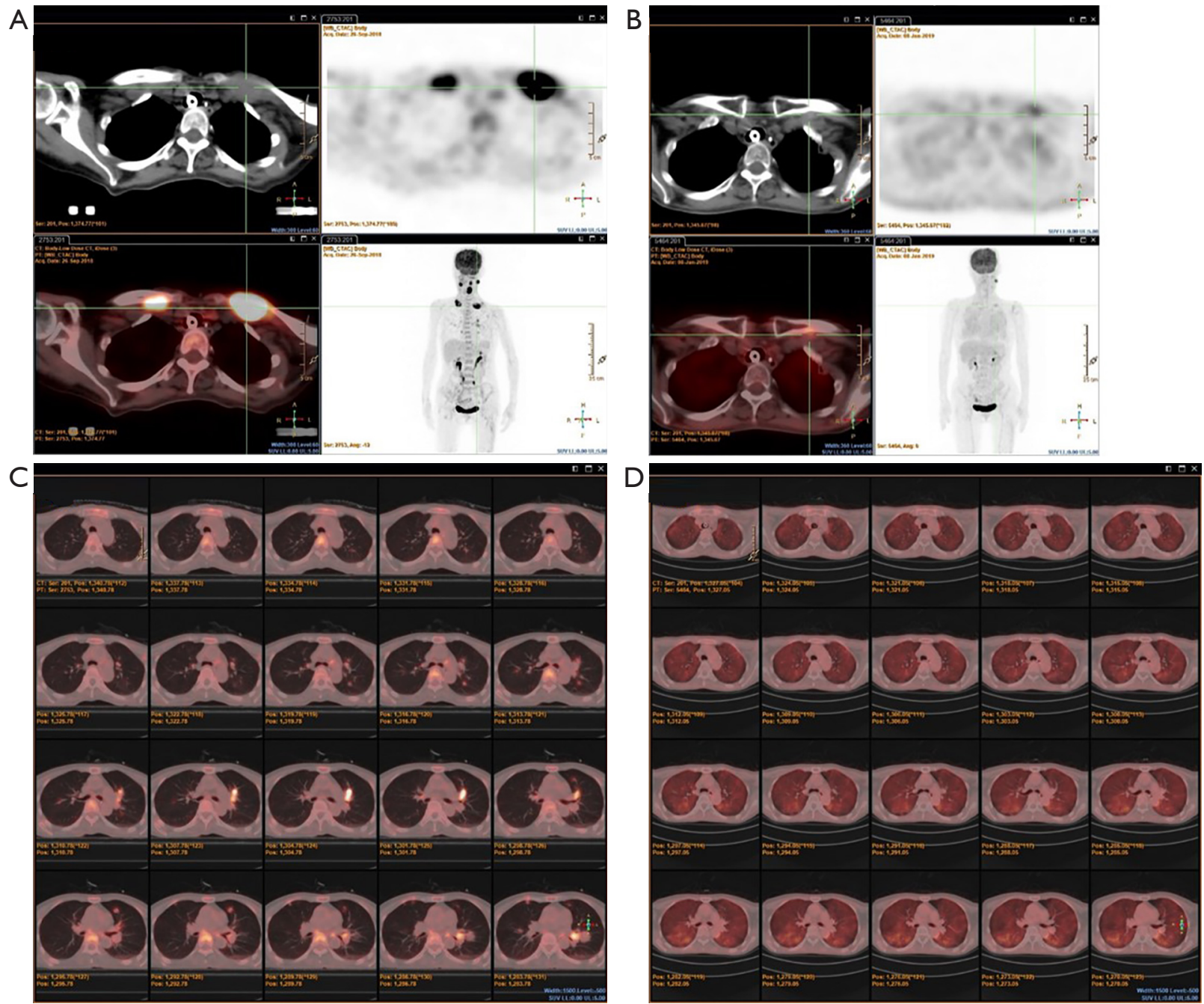

Figure 5 PET-CT scan shows different lesions during immunotherapy. (A,C) Lesions before immunotherapy were indicated by arrows; (B,D) lesions after immunotherapy were indicated by arrows. PET-CT, positron emission tomography-computed tomography.

in those with strong PD-L1 expression. We are looking forward to more results about ICI combination therapy in HNSCC in the future.

\section{Acknowledgments}

Funding: This work was supported by the National Natural Science Foundation of China (No. 81570949 to Yue He)

\section{Footnote}

Conflicts of Interest: The authors have no conflicts of interest to declare.
Etbical Statement: The authors are accountable for all aspects of the work in ensuring that questions related to the accuracy or integrity of any part of the work are appropriately investigated and resolved. Written informed consent was obtained from the patient for publication of this Case report and any accompanying images.

Open Access Statement: This is an Open Access article distributed in accordance with the Creative Commons Attribution-NonCommercial-NoDerivs 4.0 International License (CC BY-NC-ND 4.0), which permits the noncommercial replication and distribution of the article with the strict proviso that no changes or edits are made and the 
original work is properly cited (including links to both the formal publication through the relevant DOI and the license). See: https://creativecommons.org/licenses/by-nc-nd/4.0/.

\section{References}

1. Chen $\mathrm{W}$, Zheng R, Baade PD, et al. Cancer statistics in China, 2015. CA Cancer J Clin 2016;66:115-32.

2. Zeng $\mathrm{H}$, Chen $\mathrm{W}$, Zheng $\mathrm{R}$, et al. Changing cancer survival in China during 2003-15: a pooled analysis of 17 population-based cancer registries. Lancet Glob Health 2018;6:e555-67.

3. Vermorken JB, Mesia R, Rivera F, et al. Platinum-based chemotherapy plus cetuximab in head and neck cancer. $\mathrm{N}$ Engl J Med 2008;359:1116-27.

4. Ferris RL, Blumenschein G Jr, Fayette J, et al. Nivolumab for recurrent squamous-cell carcinoma of the head and neck. N Engl J Med 2016;375:1856-67.

5. Cohen EEW, Soulières D, Le Tourneau C, et al. Pembrolizumab versus methotrexate, docetaxel, or cetuximab for recurrent or metastatic head-and-neck squamous cell carcinoma (KEYNOTE-040): a randomised, open-label, phase 3 study. Lancet 2019;393:156-67.

6. Rischin D, Harrington KJ, Greil R, et al. Protocolspecified final analysis of the phase 3 KEYNOTE-048 trial of pembrolizumab (pembro) as first-line therapy for recurrent/metastatic head and neck squamous cell carcinoma (R/M HNSCC). J Clin Oncol 2019;37:6000.

7. Riley DS, Barber MS, Kienle GS, et al. CARE guidelines for case reports: explanation and elaboration document. J Clin Epidemiol 2017;89:218-35.

8. Reck M, Rodríguez-Abreu D, Robinson AG, et al. Pembrolizumab versus chemotherapy for PD-L1positive non-small-cell lung cancer. $\mathrm{N}$ Engl J Med 2016;375:1823-33.

9. Robert C, Schachter J, Long GV, et al. Pembrolizumab versus Ipilimumab in Advanced Melanoma. N Engl J Med 2015;372:2521-32.

10. Bellmunt J, de Wit R, Vaughn DJ, et al. Pembrolizumab as second-line therapy for advanced urothelial carcinoma. $\mathrm{N}$ Engl J Med 2017;376:1015-26.

Cite this article as: Tang X, Chen S, Sui Q, Li X, Liu Z, Zhu F, Ding J, Yao Y, Jiang B, He Y. Response to nivolumab combining radiotherapy and nimotuzumab in metastatic oral squamous cell carcinoma patient with strong PD-L1 expression: a case report. Ann Transl Med 2020;8(6):402. doi: 10.21037/atm.2020.02.96
11. Carbone DP, Reck M, Paz-Ares L, et al. First-line nivolumab in stage iv or recurrent non-small-cell lung cancer. N Engl J Med 2017;376:2415-26.

12. Hellmann MD, Ciuleanu TE, Pluzanski A, et al. Nivolumab plus ipilimumab in lung cancer with a high tumor mutational burden. N Engl J Med 2018;378:2093-104.

13. Shaverdian N, Lisberg AE, Bornazyan K, et al. Previous radiotherapy and the clinical activity and toxicity of pembrolizumab in the treatment of non-small-cell lung cancer: a secondary analysis of the KEYNOTE-001 phase 1 trial. Lancet Oncol 2017;18:895-903.

14. Manukian G, Bar-Ad V, Lu B, et al. Combining radiation and immune checkpoint blockade in the treatment of head and neck squamous cell carcinoma. Front Oncol 2019;9:122.

15. Motzer RJ, Penkov K, Haanen J, et al. Avelumab plus axitinib versus sunitinib for advanced renal-cell carcinoma. N Engl J Med 2019;380:1103-15.

16. Rini BI, Plimack ER, Stus V, et al. Pembrolizumab plus axitinib versus sunitinib for advanced renal-cell carcinoma. N Engl J Med 2019;380:1116-27.

17. Hodi FS, Hwu WJ, Kefford R, et al. Evaluation of immune-related response criteria and RECIST v1.1 in patients with advanced melanoma treated with pembrolizumab. J Clin Oncol 2016;34:1510-7.

18. Champiat S, Ferrara R, Massard C, et al. Hyperprogressive disease: recognizing a novel pattern to improve patient management. Nat Rev Clin Oncol 2018;15:748-62.

19. Stein S, Pishvaian MJ, Lee MS, et al. Safety and clinical activity of $1 \mathrm{~L}$ atezolizumab + bevacizumab in a phase $\mathrm{Ib}$ study in hepatocellular carcinoma (HCC). J Clin Oncol 2018;36:4074.

20. Ikeda M, Sung MW, Kudo M, et al. A phase $1 \mathrm{~b}$ trial of lenvatinib (LEN) plus pembrolizumab (PEM) in patients (pts) with unresectable hepatocellular carcinoma (uHCC). J Clin Oncol 2018;36:4076.

21. Saâda-Bouzid E, Defaucheux C, Karabajakian A, et al. Hyperprogression during anti-PD-1/PD-L1 therapy in patients with recurrent and/or metastatic head and neck squamous cell carcinoma. Ann Oncol 2017;28:1605-11. 